\title{
Análisis del impacto social y económico de la desnutrición en países como Chile, México, Ecuador y Paraguay durante el periodo 2001 al 2030
}

\section{Analysis of the social and economic impact of malnutrition in countries such as Chile, Mexico, Ecuador and Paraguay during the period 2001 to 2030}

\author{
Fatima Elisa Caballero Aguilera ${ }^{\circledR}$, Giovanna Stella Sánchez Rojas ${ }^{1} \odot$
}

\begin{abstract}
Resumen
Esta investigación presenta el impacto social y económico que genera la desnutrición en países como Chile, Ecuador, México y Paraguay en periodos comprendidos entre 2001 al 2030, la investigación surge ante la necesidad de tener un panorama de la situación de los países mencionados en cuanto a desnutrición y los efectos tanto sociales como económicos que esta genera, con el fin de plantear posibles estrategias o soluciones para lograr parte del objetivo dos de los ODS, por lo que el objetivo general del trabajo es analizar el impacto social y económico que genera la desnutrición en países como Chile, Ecuador, México y Paraguay durante el periodo del 2001 al 2030. El marco metodológico se basó en la revisión bibliográfica documental, se utilizó métodos sintético e inductivo, tuvo un nivel descriptivo, de tipo longitudinal. Se observó que la desnutrición tiene efectos en la educación, en la salud y en la productividad de las personas que la sufrieron en algún periodo de sus vidas (este periodo se da principalmente en niños menores de cinco años), se observó también que importantes costos económicos para el conjunto de la sociedad pueden ser derivados de la desnutrición debido a que la mayor probabilidad de que los niños y niñas desnutridas se enfermen aumenta considerablemente los costos en el sector salud y quienes han sufrido desnutrición infantil presentan menor capacidad de atención y aprendizaje, lo que incrementa los costos del sistema educativo, en cuanto a la productividad, se refiere a la pérdida de capital humano que se genera para una sociedad, debido al menor nivel educativo que alcanzan las personas que sufren desnutrición, además de las muertes que causa la desnutrición, lo que se traduce en pérdida de capacidad productiva. Finalmente se llegó a la conclusión de que los Estados, de cada país analizado, deben promover políticas públicas que permita a la población acceder a una dieta saludable y, además de eso que dicha población conozca la importancia que tiene el consumo de los nutrientes necesarios, principalmente en niños menores de cinco años, considerando que los efectos y los costos se distribuyen a lo largo del ciclo de la vida, esto con el fin de disminuir el impacto y el costo que genera en la salud, en la educación y en la productividad.
\end{abstract}

1 Universidad Nacional de Asunción, Facultad de Ciencias Económicas. San Lorenzo, Paraguay.

Correspondencia a: fcaballero973@alumnos.eco.una.py

Recibido:

17 de setiembre de 2021

Aceptado:

5 de noviembre de 2021

Doi:

https://doi.org/10.54549/ky.6e.2021.89

Cc)
Artículo publicado en acceso
abierto bajo la Licencia Creative
Commons.
Cita:
Caballero Aguilera, F. E.,
Sánchez Rojas, G. S. (2021).
Análisis del impacto socialy
económico de la desnutrición
en países como Chile, México,
Ecuadory Paraguay durante
el periodo 2oo1 al 2o3o. Kera
Yvoty: reflexiones sobre la
cuestión social, 6(número
especial), 89-97. https://doi.
org/10.54549/ky.6e.2021.89


Caballero Aguilera y Sánchez Rojas. Análisis del impacto social y económico de la desnutrición en países como Chile, México, Ecuador y Paraguay durante el periodo 2001 al 2030

Palabras clave: desnutrición, impacto, social, económico.

\begin{abstract}
This research presents the social and economic impact generated by malnutrition in countries such as Chile, Ecuador, Mexico and Paraguay in periods from 2001 to 2030, the research arises from the need to have a picture of the situation of the countries mentioned in terms of malnutrition and the social and economic effects that it generates, in order to propose possible strategies or solutions to achieve part of goal two of the SDGs, so the general objective of the work is to analyze the social and economic impact generated by malnutrition in countries such as Chile, Ecuador, Mexico and Paraguay during the period from 2001 to 2030. The methodological framework was based on the documentary literature review, synthetic and inductive methods were used, had a descriptive level, longitudinal type. It was observed that malnutrition has effects on education, health and productivity of people who suffered from it at some period of their lives (this period occurs mainly in children under five years of age), it was also observed that significant economic costs for society as a whole can be derived from malnutrition because the greater probability of malnourished children getting sick increases considerably the costs in the health sector and those who have suffered child malnutrition have less attention and learning capacity, which increases the costs of the education system, in terms of productivity, refers to the loss of human capital that is generated for a society, due to the lower level of education reached by people suffering from malnutrition, in addition to the deaths caused by malnutrition, which translates into loss of productive capacity. Finally, it was concluded that the States, in each country analyzed, should promote public policies that allow the population to access a healthy diet and, in addition to that, the population should know the importance of the consumption of the necessary nutrients, mainly in children under five years of age, considering that the effects and costs are distributed throughout the life cycle., this in order to reduce the impact and cost it generates in health, education and productivity.
\end{abstract}

Keywords: malnutrition, impact, social, economic.

\section{Introducción}

Esta investigación busca analizar el impacto social y económico que tiene la desnutrición en países como Chile, Ecuador, México y Paraguay.

La alimentación está reconocida a nivel internacional como un derecho humano, mientras que el acceso físico y económico a alimentos nutritivos con el fin de satisfacer necesidades alimentarias y llevar una vida sana y activa es llamado seguridad alimentaria y nutricional (Imas et al., 2019).

La Agenda 2030 sobre el Desarrollo Sostenible fue aprobada por la ONU en 2015, esta agenda se presenta como una oportunidad para que los países puedan tener un camino y logren mejorar el bienestar de toda su población. La Agenda tiene 17 Objetivos de Desarrollo Sostenible; este trabajo sigue una línea en torno al objetivo 2: poner fin al hambre o hambre cero. El objetivo 2 se divide en ocho metas, la de nuestro interés es la meta $\mathbf{2 . 2}$ que busca poner fin a todas las formas de malnutrición, incluso para antes del 2030.

La CEPAL y el Programa Mundial de Alimentos (PMA) realizaron estudios sobre el costo del hambre en Centroamérica, los países andinos y el Paraguay, estos estudios permitieron demostrar que la desnutrición, que se mide como bajo peso para la edad, tiene efectos en los costos de salud, educación y productividad (Martínez \& Fernández, 2009 y 2007 citado por Fernández et al., 2017), por lo que es importante tener en cuenta a la desnutrición y los efectos que esta causa para que los países puedan ser eficientes en lo que al gasto público se refiere.

La importancia de esta investigación proviene de la necesidad de tener un panorama de la situación de desnutrición 
de cada uno de los países estudiados y los efectos que esta causa, con el fin de plantear posibles soluciones para así lograr parte de la meta 2.2 del objetivo 2 de los ODS.

En ese sentido, planteamos la siguiente interrogante ¿Cómo impacta social y económicamente la desnutrición en países como Chile, Ecuador, México y Paraguay en el periodo 2001 al 2030? De esta manera, buscamos mostrar que los costos generados por la desnutrición no se refieren solo a un año determinado o a corto plazo, sino que tiene efectos y sigue generando costos también a largo plazo.

\section{Objetivo general}

Analizar el impacto social y económico de la desnutrición en Chile, Ecuador, México y Paraguay en el periodo 2001 al 2030.

\section{Metodología}

La investigación se basó principalmente en la modalidad bibliográfica documental, mediante la utilización de libros, publicaciones, documentos y base de datos, obtenidos de sitios oficiales como FAO, CEPAL, Banco Mundial, etc.

La investigación tiene un nivel descriptivo ya que se describe la manera en que la desnutrición causa los efectos y costos en la salud, la educación y la productividad.

El tipo de estudio de la investigación según el tiempo es longitudinal, se analiza los efectos y costos de la desnutrición en diferentes periodos de tiempo, específicamente, para el análisis de la prevalencia de la desnutrición, el periodo analizado es desde el 2001 al 2018 para los cuatro países analizados. En cuanto a los efectos y costos descritos, el análisis de Paraguay corresponde al año 2005, de Ecuador y México corresponden al año 2014, además, para estos dos últimos se incluye una estimación para el periodo 2014 al 2030.

Los métodos utilizados fueron el sintético y el inductivo, puesto que primeramente se parte de los efectos y costos en la salud, en la educación y en la productividad, por separado y luego se procede a presentar los costos totales de la desnutrición para cada uno de los países seleccionados.

\section{Resultados}

En el estudio de Fernández et al. (2017), el concepto de malnutrición incluye, tanto a la desnutrición que, tiene en cuenta los indicadores de bajo peso al nacer, la insuficiencia de peso, el rezago de talla, la baja relación peso/talla y el déficit de micronutrientes; incluye también al sobrepeso y la obesidad, además, afirma que la malnutrición impacta de manera intergeneracional debido a que los efectos pueden manifestarse a lo largo del ciclo de vida y de distintas maneras, lo que hace que los problemas a los cuales se enfrentan los países sean de elevada complejidad.

Fernández et al. (2017), enumera los principales factores que afectan y determinan que la desnutrición sea un problema social y de salud pública; estos factores se pueden agrupar según sus características, por ejemplo, encontramos a las medioambientales, que tiene relación con el entorno en que vive una familia o un individuo, también, las socio-culturaleseconómicos, que hacen referencia a lo relacionado con la pobreza y desigualdad, escolaridad y pautas culturales, salarios, nivel de empleo, etc.; por último, los políticos-institucionales, estos incluyen a los programas gubernamentales y las políticas orientados a resolver los problemas alimentario-nutricionales de la población.

Una estimación hecha antes de la pandemia del COVID-19 mostró que alrededor de 690 millones de personas, lo que representa el $8,9 \%$ de la población mundial, estaban subalimentadas, y la tendencia sigue al alza desde el 2014, si la tendencia continúa de esta manera, se estima que para el año 2030, el número de personas subalimentadas superará los 840 millones. Por lo que el mundo no está en vías de lograr el objetivo del hambre cero, esto incluso sin tener en cuenta los 
Figura 1. Costo (en USD) de una dieta energética mínima y una dieta saludable..

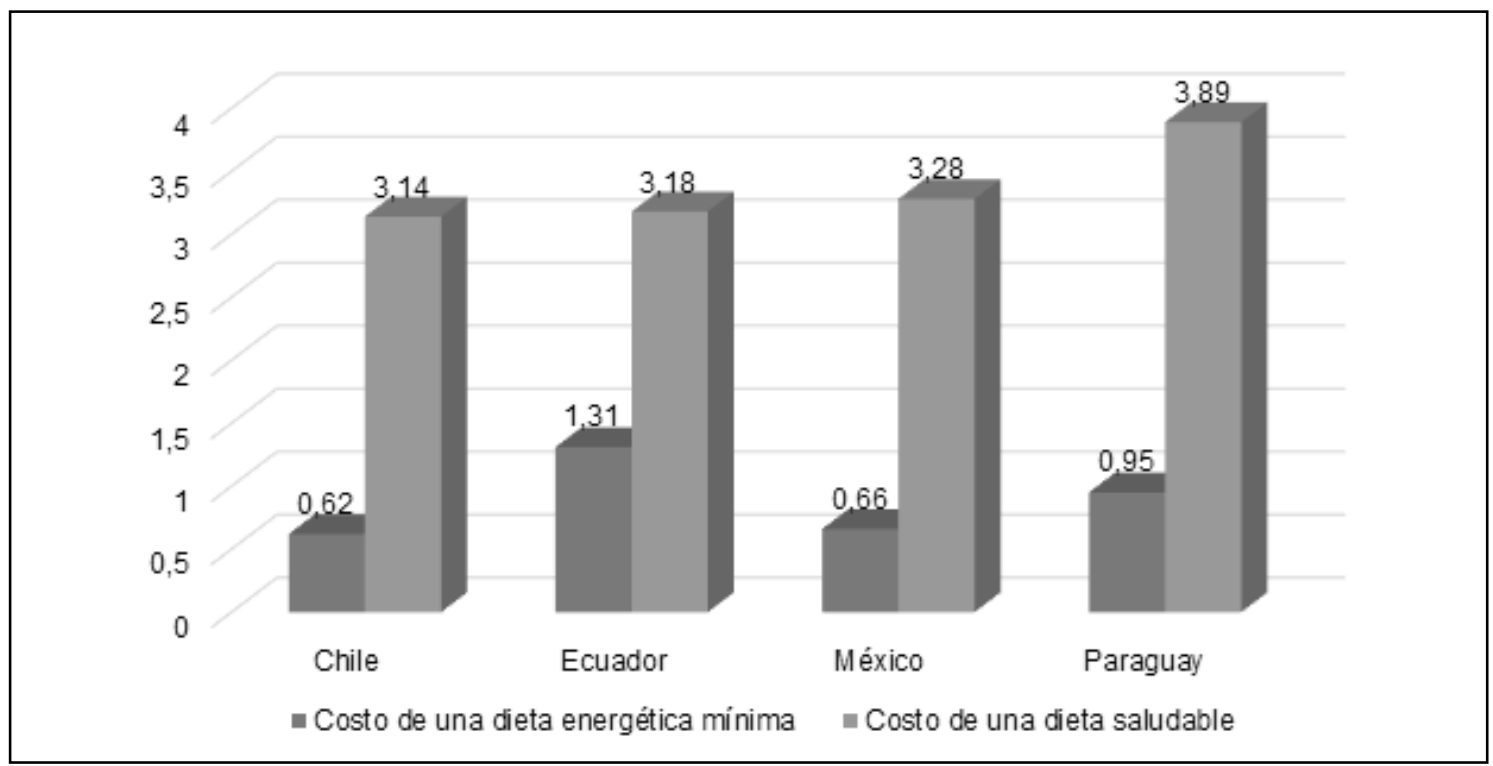

Fuente. Elaboración propia, con base en datos de FAO, FIDA, OMS, PMA y UNICEF, 2020.

efectos de la pandemia del COVID-19 que, según estimaciones preliminares, podría sumar entre 83 y 132 millones de personas subalimentadas (FAO et al., 2020).

En el mismo informe se menciona que, de continuar los hábitos actuales de consumo de alimentos, los costos sanitarios relacionados con la dieta, que derivan de la mortalidad y las enfermedades no transmisibles relacionadas con la alimentación, asciendan a más de1,3 billones de USD al año para 2030. Se estima también que los costos sociales relacionados con la dieta, que tienen relación con las emisiones de gases de efecto invernadero asociadas a los hábitos actuales de alimentación, llegarán a más de 1,7 billones de USD al año para 2030.

A raíz de los Objetivos de Desarrollo Sostenibley de los efectos tanto económicos como sociales que tiene la desnutrición y, además, teniendo en cuenta que tanto los costos sanitarios como los costos sociales están ampliamente subestimados, los países luchan para erradicar la desnutrición en sus respectivas poblaciones.

Se analizan los costos de las canastas de alimentos en cada uno de los países ya mencionados, como también la población de cada país que no puede acceder a esas canastas, en la Figura 1 y 2 observamos estos datos, respectivamente.

Paraguay destaca por tener el costo de dieta saludable más elevado de entre los cuatro países que estamos analizando, en el gráfico 1 se observa que a la población paraguaya le cuesta USD 3,89 acceder a una dieta saludable, la cual, según FAO et al. (2020), este costo representa el 66,3\% del gasto promedio en alimentación, en un principio este costo es asequible ${ }^{1}$, sin embargo, considerando los ingresos medios de la población, el $18 \%$ no puede acceder a una dieta saludable. Por otro lado, la dieta básica en cuanto a energía, en Paraguay, tiene un costo de USD 0,95,

\footnotetext{
1 La dieta resulta inasequible cuando su costo supera el $63 \%$ de los ingresos medios en un determinado país. Se considera que el $63 \%$ representa la parte de los ingresos medios que se puede destinar a alimentación (Herforth, A., Bai, Y., Venkat, A., Mahrt, K., Ebel, A. y Masters, W.A, 2020. Cost and affordability of healthy diets across and within countries, citado por FAO et al., 2020)
} 
Figura 2. Población que no puede acceder a una dieta mínima en cuanto a energía y a una dieta saludable (en \%).

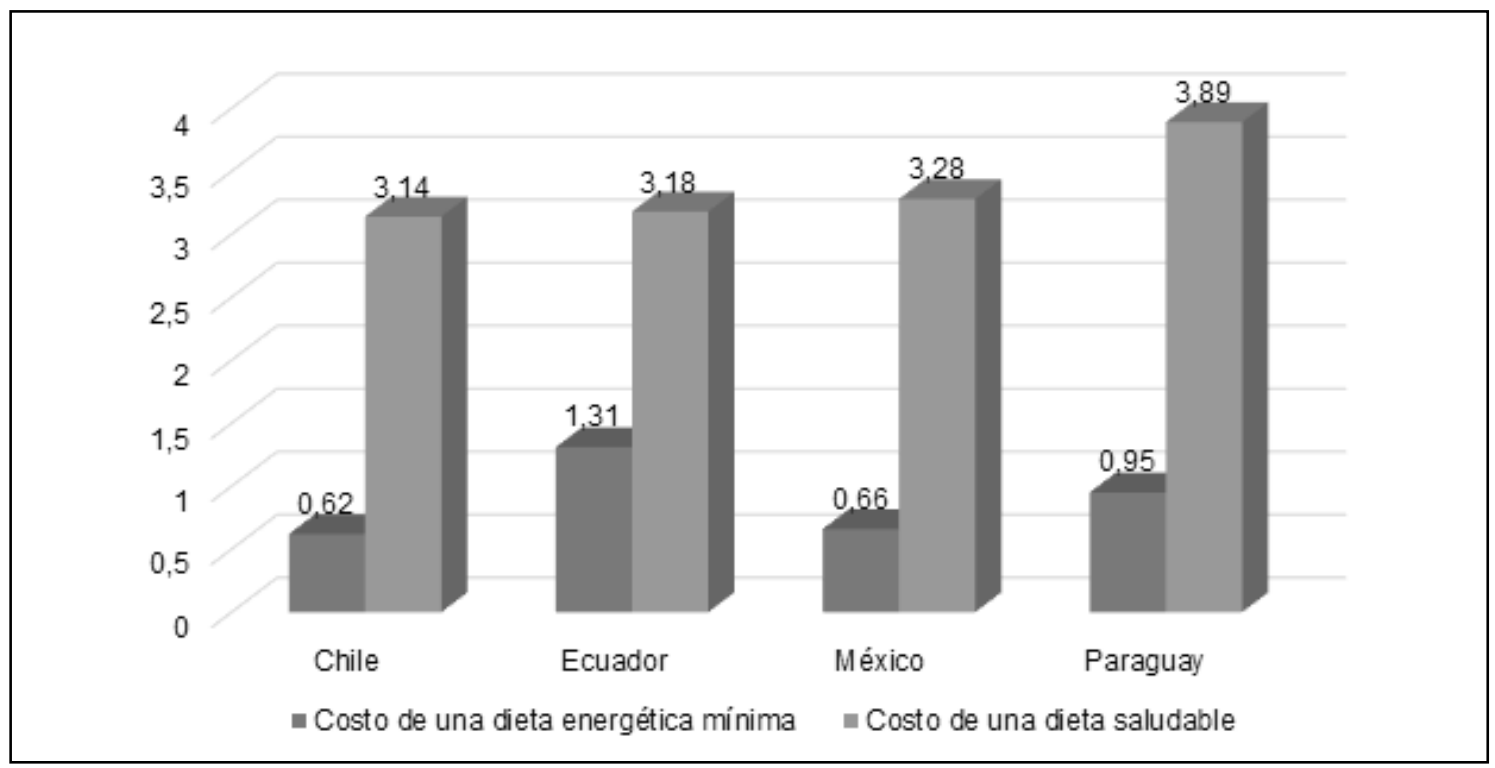

Fuente. Elaboración propia, con base en datos de FAO, FIDA, OMS, PMA y UNICEF, 2020.

representando el $16,2 \%$ de los gastos medios en alimentos de la población, por lo que una mayor proporción puede acceder a este tipo de dieta y solo el o,6\% de la población paraguaya no puede acceder a ella.

En cuanto a Chile, Ecuador y México, los costos observados en el gráfico 1 representan el 50,4\%; 74,2\% y $41,6 \%$ de los costos promedios en alimentación, respectivamente; teniendo en cuenta los ingresos medios de la población de cada uno de los países mencionados, las dietas saludables en Chile y en México se consideran asequibles. En el gráfico 2 se observa que solo el 1,8\% de la población chilena no puede acceder a una dieta saludable y el $17,2 \%$ de la población mexicana se encuentra en esta misma situación; el costo de la dieta saludable en Ecuador, por su parte, representa una proporción relativamente elevada de los costos promedios en alimentación de este país, es considerado inasequible $\mathrm{y}$, como se muestra en el gráfico 2 , el $18,2 \%$ de la población de Ecuador no puede acceder a este tipo de dieta. Observemos ahora, en el gráfico 1 , los resultados en cuanto a la dieta energética básica, estos costos representan el 10\%;30,5\% y $8,4 \%$ de los costos promedios en alimentación en Chile, Ecuadory México, respectivamente, el costo de esta dieta mínima en cuanto a energía resulta más asequible que la dieta saludable, por lo que solamente el $0,2 \%$ de la población chilena, el 30,5\% de la población ecuatoriana y el $8,4 \%$ de la población mexicana, no puede acceder a ella (FAO et al., 2020).

Como hemos visto, una buena parte de la población de cada uno de los países no puede acceder a una dieta saludable, este tipo de dieta ayuda a reducir el riesgo de cualquier forma de malnutrición y, en particular, la desnutrición (FAO et al., 2020).

El hecho de que un bajo porcentaje de la población no pueda acceder a la dieta mínima en cuanto a energía, no significa que el resto sí lo haga, aunque tengan las posibilidades económicas para hacerlo, no siempre es el caso, ya sea porque resulta más barato consumir alimentos que no aportan ni siquiera el valor nutricional mínimo 
Caballero Aguilera y Sánchez Rojas. Análisis del impacto social y económico de la desnutrición en países como Chile, México, Ecuador y Paraguay durante el periodo 2001 al 2030

o porque simplemente desconocen los efectos de consumir este tipo de alimentos, por lo que una parte de la población de cada uno de los países analizados está desnutrida, estos resultados se presentan a continuación, en la Figura 3.

En la Figura 3 se observa que desde el año 2001 los países vienen trabajando para disminuir la prevalencia de la desnutrición, el país que más destaca es Ecuador, el cual, en el 2002, más del $\mathbf{2 2} \%$ de la población estaba desnutrida, pudiendo lograr una tendencia a la baja y para el 2018 la cifra llegó a significar el 9\% de la población; Paraguay, por su parte, tuvo una prevalencia de la desnutrición que representaba el 10,6\% de la población en 2001, manteniendo una tendencia relativamente constante, la prevalencia de desnutrición comienza a disminuir en el año 2009 y la cifra más baja para este país fue de $7,7 \%$ en el 2014, año en el cual comenzó a aumentar de nuevo, llegando al 8,8\% para el 2018; por otro lado, en México la prevalencia de desnutrición representaba el 3,3\% de la población en el año 2001, con una tendencia más bien al alza, para el 2018 la cifra llegó a representar al 7,1\% de la población mexicana; el último país del análisis es Chile, este país presenta una tendencia relativamente establey, tanto en el 2001 como en el 2018, la prevalencia de desnutrición fue de $3,5 \%$.

Comoyaseadelantóenlaintroducción de este trabajo, la desnutrición tiene efectos en la educación, la salud y la productividad de las personas que sufrieron desnutrición en algún periodo de sus vidas (este periodo se da principalmente en menores de 5 años), a nivel educativo, el desempeño escolar se ve afectado debido a las limitaciones en la capacidad de aprendizaje que se asocia a las restricciones en el desarrollo cognitivo y a los déficits que generan las enfermedades, es decir, existe mayores probabilidades de deserción, ingreso tardío y repitencia, lo que, eventualmente, se traduce como bajo nivel educativo (Martínez \& Fernández, 2009).

Importantes costos económicos para el conjunto de la sociedad pueden ser derivados de la desnutrición y sus efectos en salud y educación, ya que la mayor probabilidad de que los niños y niñas desnutridas se enfermen aumenta

Figura 3. Prevalencia de la Desnutrición (\% de la población).

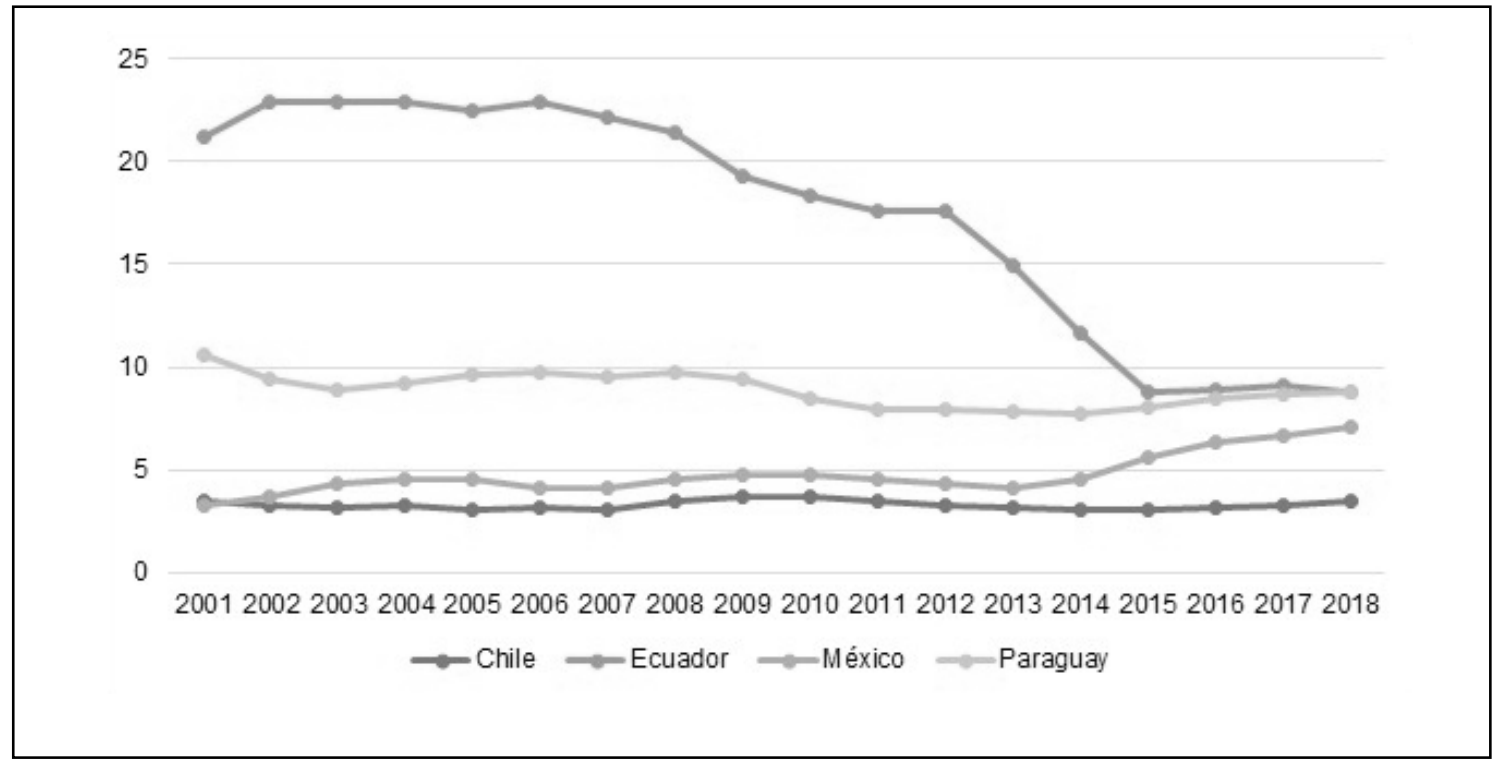

Fuente. Elaboración propia, con datos del Banco Mundial, 2021. 
considerablemente los costos en el sector salud y, quienes han sufrido desnutrición infantil, presentan menor capacidad de atención y aprendizaje, lo que incrementa los costos del sistema educativo. En cuanto a productividad, se refiere a la pérdida de capital humano que se genera para una sociedad, debido al menor nivel educativo que alcanzan las personas que sufren desnutrición, además de las muertes que causa la desnutrición, lo que se traduce en pérdida de capacidad productiva (Martínez \& Fernández, 2009).

A continuación, se describirán los costos a los que cada uno de los países incurre debido a la desnutrición.

Martínez y Fernández (2009), presentan que, en 2005, el costo por menor desnutrido, en Paraguay, fue de USD 1004 (35 millones de dólares en total), representó el 41,2\% del gasto público en salud yequivale al o, $5 \%$ del PIB de dicho país.

En cuanto a Ecuador y México, Fernández et al. (2017), estiman que en 2014 el costo total en salud asociado a desnutrición fue de 44 millones de dólares y más de 900 millones de dólares, respectivamente; en estos países, estos costos representan el o,04\% del PIB de Ecuador y 0,07\% del PIB de México, y equivalen al $2,42 \%$ y $2,61 \%$ del gasto social en salud, respectivamente.

Noseestiman costos para Chiledebido a que se considera que la desnutrición en dicho país está erradicada.

El costo adicional del sistema educativo fue de 833 mil dólares, en Paraguay, en 2005, debido a los educandos extra (más de 2.00o) que repitieron debido a la desnutrición; el $96 \%$ de este costo fue para la educación primaria y el $4 \%$ para la educación secundaria, este costo representa el $0,28 \%$ del gasto público en educación y el o,or\% del PIB del país (Martínez \& Fernández, 2009).

En 2014, los repitentes extra que se habrían generado por la desnutrición, incrementaron en 27 millones de dólares el costo del sistema educativo de Ecuador (más de 26.000 repitentes) y 151 millones de dólares en México (más de 86.000 repitentes); más del $36 \%$ del costo fue destinado a la educación primaria y más del $63 \%$ a la educación secundaria de Ecuador, mientras que, en México, más del 11\% de los costos fue para educación primaria y más del $88 \%$ a la educación secundaria. Estos costos representan el o,027\% y el o,012\% del PIB en Ecuador y México, respectivamente; en cuanto al gasto social en educación, los costos equivalen al $0,59 \%$ en Ecuador $\mathrm{y}$ 0,34\% en México (Fernández et al., 2017).

En cuanto a los efectos y costos en la productividad, se ve reflejado de manera negativa en la capacidad productiva del país, resultado de la mayor prevalencia de mortalidad; en Paraguay, alrededor de 44 mil muertes fueron causados por la desnutrición, esto equivale a aproximadamente 49 millones de horas de trabajo perdidas, esto es un poco más de $1,31 \%$ de las horas trabajadas por la población económicamente activa (PEA). Para el 2005 se estima que se generaron pérdidas de aproximadamente 73 millones de dólares, debido a la menor escolaridad, lo que equivale a 3,1\% del gasto social del año, $6,4 \%$ del gasto público en educación y $1 \%$ del PIB. Por otro lado, debido a las muertes, se estima que la pérdida de productividad fue de aproximadamente 40 millones de dólares, esto es un 2,54\% del PIB; en el mismo año, el país habría sido 1,6\% más productivo en ese año de no ser por los niveles de desnutrición sufridos en las décadas anteriores (Martínez \&Fernández, 2009).

Para Ecuador y México, la productividad por menor escolaridad generó pérdidas de $\mathbf{1 . 5 2 8}$ millones de dólares y 14.662 millones de dólares, respectivamente, mientras que el costo o pérdida por mortalidad fue de 1.001 millones de dólares en Ecuador y 5.796 millones de dólares en México, lo que equivalen a $2.51 \%$ y $1,58 \%$ del PIB, respectivamente; $31,31 \%$ y $14,83 \%$ del gasto social total para ambos países (Fernández et al., 2017).

Según lo mencionado, y las fuentes consultadas, debido a la desnutrición, el 
Caballero Aguilera y Sánchez Rojas. Análisis del impacto social y económico de la desnutrición en países como Chile, México, Ecuador y Paraguay durante el periodo 2001 al 2030

costo total de Paraguay en 2005 fue de 149,2 millones de dólares, en 2014 el costo total de Ecuador fue de 2.599 millones de dólares y de México 24.516 millones de dólares, México presenta el mayor costo total absoluto por desnutrición, esto obedece al mayor tamaño de su población y economía.

Fernández et al. (2017), estiman que para el 2030, el costo en salud para

Ecuador será de 0,72 millones de dólares, expresado en costo anual equivalente (CAE), a una tasa de descuento de $3 \%$ y representará el $0,040 \%$ del gasto público en salud, en México, el costo en salud será de 3,9 millones de dólares (CAE) y representará el o,o11\% del gasto social en salud.

En cuanto al costo que genera la repitencia en el sistema escolar, Fernández et al. (2017), estiman que, en Ecuador, para el 2030 será de o,8 millones de dólares, CAE a una tasa de descuento del $3 \%$, que representa el o,016\% del gasto público en educación. Para México, el costo será de 2,8 millones de dólares, CAE a una tasa del 3\% de descuento, que representa el o,oo6\% del gasto público en educación.

Por último, el costo en productividad asociado a desnutrición en Ecuador será de o,43 millones de dólares (CAE) y representará el $0,005 \%$ del gasto público social, México tendrá un costo de 0,95 millones de dólares (CAE), equivalente al o,oo1\% del gasto social público (Fernández et al., 2017).

No se presentan los costos futuros de Paraguay debido a la falta de estimaciones para el periodo de interés.

\section{Discusión y Conclusiones}

Los costos estimados en cuanto a productividad son aparentemente bajos, sin embargo, si tenemos en cuenta el periodo de tiempo de esa estimación (de 2014 a 2030) la población estudiada aún no se incorporaría totalmente a la fuerza laboral, en el estudio de Fernández et al. (2017) cuando considera el tiempo necesario para que la población se integre a la fuerza laboral (de 2014 a 2078) estos costos se elevan significativamente y no solo los de productividad, también los costos en salud y en educación.

Es importante destacar que las estimaciones se hicieron sin tener en cuenta los efectos de la pandemia del COVID-19 por lo que es elevada la probabilidad de que los costos sean mayores.

Es necesario que los países fortalezcan las instituciones que pueden tener efecto directo sobre la población desnutrida, además de fortalecerlas, deben tener un trabajo en conjunto para que esta problemática sea erradicada, estas instituciones son los referidos al sistema de salud, el sistema educativo y el sistema laboral, que son los sectores principales afectados por la desnutrición.

Los Estados deben promover políticas públicas para que la población entera acceda a dietas saludables, pero no basta con que la dieta sea asequible, la población debe estar al tanto de la importancia de consumir los nutrientes necesarios, principalmente para los niños menores de cinco años, ya que los datos muestran que incluso cuando solo una pequeña parte de la población no puede acceder a una dieta, al menos, básica en cuanto a energía; de todas formas la prevalencia de la desnutrición es relativamente más elevada de lo que se esperaría considerando que muy pocos no pueden acceder a ese tipo de dieta, el canal de información podría ser a través de las escuelas o programas sociales, además, la población debe estar también al tanto del derecho a la alimentación.

FAO et al. (2020), recomienda que los estados creen entornos favorables para las dietas saludables y sostenibles en lugares de trabajo, escuelas, hospitales y en las instituciones públicas.

Imas (2019), recomienda que el crecimiento económico debe ir acompañado de políticas redistributivas a fin de disminuir la brecha entre pobres y ricos.

Todo esto con el fin de lograr la erradicación de la malnutrición, específicamente de la desnutrición y evitar así los costos que dicha problemática 
genera, teniendo en cuenta que los efectos y los costos se distribuyen a lo largo del ciclo de vida.

Cabe mencionar que un niño sin hambre no significa que sea un niño bien nutrido, los problemas causados por la desnutrición y los causados por el sobrepeso y la obesidad, pueden coexistir en la misma población, lo que se conoce como la doble carga de la malnutrición se traduce en costos incluso mayores que los analizados en este trabajo.

\section{Referencia}

Banco Mundial. (2021). Prevalencia de desnutrición (\% de la población) Paraguay, Chile, Ecuador. https://datos. bancomundial.org/indicador/SN.ITK.

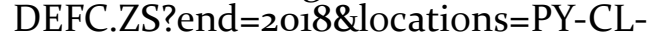
EC-MX\&start=2001\&view=chart\&ye $\mathrm{ar}=2018$

FAO, FIDA, PMA, \& UNICEF. (2020). El estado de la seguridad alimentaria y la nutrición en el mundo 2020. Transformación de los sistemas alimentarios para que promuevan dietas asequibles y saludables. FAO. https://doi.org/10.406o/cag692es
Fernández, A., Martínez, R., Carrasco, I., \& Palma, A. (2017). Impacto Social y Económico de Malnutrición: Modelo de análisis y estudio piloto en Chile, el Ecuador y México. CEPAL. Naciones Unidas. http://www.codajic.org/sites/ www.codajic.org/files/Impacto $\% 20$ social $\% 20 y \% 20$ econ $\% \mathrm{C}_{3} \% \mathrm{~B}_{3}$ mico $\% 20$ de\%2ola\%2omalnutrici $\% \mathrm{C}_{3} \% \mathrm{~B}_{3} \% 20$. pdf

Imas, V. J., Benítez, G., Serafini, V., Zavattierro, C., \& García, L. (2019). Seguridad y Soberanía Alimentaria en Paraguay. Sistema de indicadores y línea de base (Resumen). CADEP. https://www.conacyt.gov.py/sites/ default/files/upload_editores/u294/ seguridad_sober ania_alimentaria_ cadep.pdf

Martínez, R., \& Fernández A. (2009). El costo del hambre: Impacto social y económico de la desnutrición infantil en el Estado Plurinacional de Bolivia, el Ecuador, Paraguay y el Perú. División de Desarrollo Social. CEPAL, Naciones Unidas. Santiago. https:// repositorio.cepal.org/bitstream/ handle/11362/39306/1/LCW26o_es.pdf

\section{Sobre los Autores}

Fátima Elisa Caballero Aguilera

Estudiante del octavo semestre de la Carrera de Economía en la Facultad de Ciencias Económicas de la Universidad Nacional de Asunción.

\section{Giovanna Stella Sánchez Rojas}

Estudiante del octavo semestre de la Carrera de Economía en la Facultad de Ciencias Económicas de la Universidad Nacional de Asunción y miembro activo del Club de Debate Económico de la UNA. 